\title{
A model study of the daylight and energy performance of rooms adjoining
} an atrium well

\author{
Jiangtao $\mathrm{Du}^{1,{ }^{*}}$, Steve Sharples ${ }^{2}$, Neil Johnson ${ }^{1}$ \\ ${ }^{1}$ School of Architecture, University of Sheffield, Sheffield, UK \\ ${ }^{2}$ School of Architecture, University of Liverpool, Liverpool, UK \\ *Corresponding author. Tel: +44 7892841534, Fax: +44 114222 0315, E-mail: jiangtao.du@yahoo.co.uk
}

\begin{abstract}
Daylight has been regarded as a significant environmental advantage of atrium buildings because the natural light can illuminate potentially dark core areas and decrease energy consumption. This study has investigated the average daylight factors (overcast sky conditions) and annual lighting energy load (real weather conditions of Sheffield, UK) in adjoining spaces to assess the fundamental daylight performance and energy performance in an atrium model. Radiance and Daysim (based on Radiance algorithm) were the tools to simulate the daylighting and lighting energy use. A comparison of the measurement and simulation showed the validation of the basic Radiance simulation in the model. In terms of the well façades (decided by the ratio of window area to solid wall area) and well surface reflectance, the variations of daylight level and annual electrical lighting use in the adjoining rooms have been analysed and some design strategies for supporting preliminary design decisions are presented. Only the square atrium model and relatively simple climate conditions have been considered in the investigation.
\end{abstract}

Keywords: Atrium Building, Daylight Performance, Lighting Energy Saving, Simulation

\section{Introduction}

Daylight use in an atrium is particularly beneficial as the atrium well can allow natural light to reach potentially dark core areas in adjoin rooms, decrease energy use by artificial lighting and improve the indoor environment on psychological and ergonomic grounds. In general, the daylight levels in the adjoining rooms are directly influenced by the vertical daylight levels on the well facade and the room properties (size and surface reflectances) [1, 2]. A linear relationship between the vertical daylight factor at the facade and the average daylight factor in the room with a specific dimension and reflectance can be given by a theoretical expression [3]. It has been found that well geometries and surface reflectances have a direct effect on the decay of vertical daylight levels across the well wall in atria [4]. Well geometry can be quantified in terms of the well index (WI) [1], equation (1) which is a function of well length (1), width (w) and height (h) (Fig. 1):

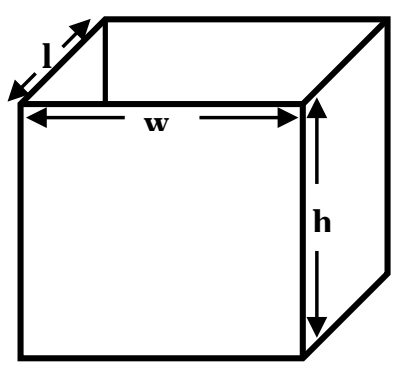

Fig. 1. Atrium geometry and WI definition [1].

$$
W I=\frac{h(w+l)}{2 w l}
$$


Two measurements using scale models $[5,6]$ pointed out that changing the proportion of glazing or opening areas between well and adjacent spaces could be a practical solution to the imbalance of light flux received at the top and bottom of the atrium walls and adjoining spaces. A common rule was suggested: small glazing areas on the top floor and the fully gazing areas on the ground floor. Nevertheless, a recent study [7] based on Radiance simulation indicated that increasing well surface reflectances had little impact on the daylight level of full glazed rooms at ground floor because of the lack of solid reflecting surfaces. This demontrates the complexities of refleted light in atria. It is still essential to carry out more investigations on daylighting performance of rooms in atria with various facade systems. Moreover, the lighting enegy saving under the conditions of daylight use in atria is another significant toipc to be studied. A field measurement [8] in two large atria showed that daylighting illumination can displace $46 \%$ and around $15 \%$ of annual lighting consumptions through introducing two different lighting control systems respectively. By providing sufficient daylighting illumination, the daylight use in atria displaces the artificial lighting load in the adjacent spaces, which is regarded as one of the most important and hardest-toachieve objectives of atrium environmental design [2].

This study investigated the daylight performance and energy performance (annual lighting use) of adjoining rooms in a five-story atrium model with various façade systems and well reflectances. Radiance and DaySim (Radiance-based package) were used in the calculations of daylight factor and annual lighting load respectively. Firstly, a comparison between model measurements and Radiance simulations was undertaken to validate the basic Radiance outputs. Next, more Radiance simulations were carried out to display the impact of façade configurations and well reflectances on the average daylight factors in rooms. Thirdly, the lighting energy consumption of rooms in the atrium model was considered. Finally, some design strategies have also been suggested.

\section{Comparisons of simulation and measurement}

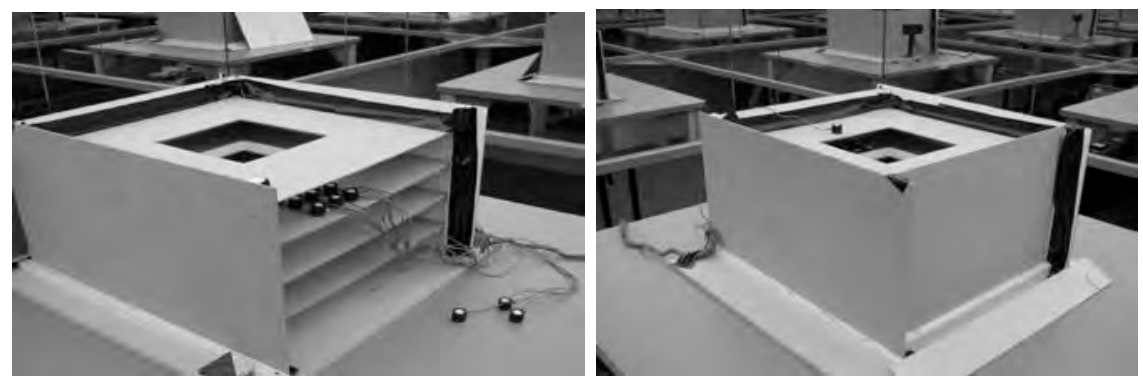

Fig. 2. Physical atrium model in a mirror box artificial sky.

A physical atrium building model was tested in a mirror box artificial sky that reproduced a CIE standard overcast sky (Fig.2). The measured data were compared with simulated data from Radiance. The scale building model had an atrium well and adjoining spaces. In the centre of the building, the well had a square plan of $200 \mathrm{~mm} \times 200 \mathrm{~mm}$ whilst the whole building had a square plan of $500 \mathrm{~mm} \times 500 \mathrm{~mm}$. With a height of $350 \mathrm{~mm}$ the atrium well had a WI value of 1.75 , which represents a medium atrium. Four-storey adjoining spaces were set around the well and the height and the depth of each side room at each floor were $70 \mathrm{~mm}$ and $150 \mathrm{~mm}$ respectively. There were two different façade types: rooms with unobstructed windows and rooms with balconies on the border of the well (balcony height $=1 / 3$ room height). The well floor had been given three different surface reflectances: low (black), medium (grey) and high (white). 
For models with balconies, the comparisons of measured and simulated average daylight factors (ADF) in rooms are indicated in Fig.3. It can be seen that, generally, the simulations are close to the measurements at the ground, $1^{\text {st }}$ and $2^{\text {nd }}$ floors. However, the measured data are underestimated by the simulations at the top floor, which is dominated by direct sky light. Taking the measurements as reference, the percentage differences of simulations are $18.5 \%$ (maximum), 0.06\% (minimum) and 9.5\% (average) respectively.
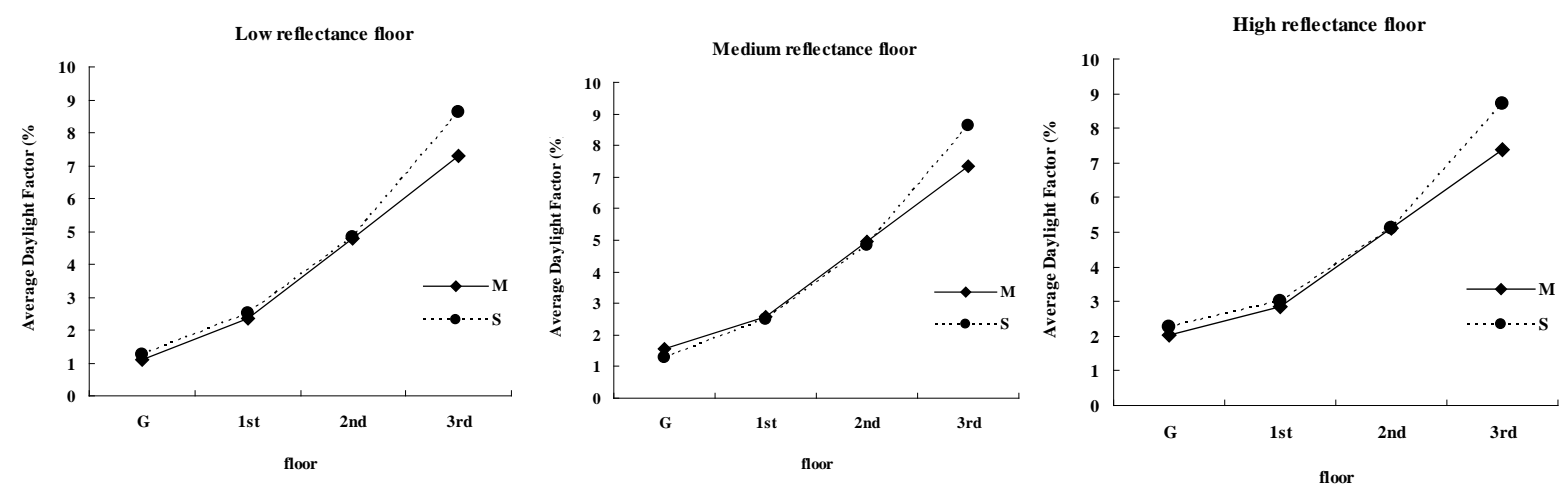

Fig. 3. Comparison of measured (M) and simulated (S) average daylight factors at each floor in a four-story atrium model with balconies.

For models with open facades, Fig. 4 shows the comparisons of measured and simulated ADF in the adjoining rooms. The models without balconies have a very similar form of variation of average daylight level to the models with balconies: the top floor has a divergence between the two values; other floors have achieved a good agreement. Likewise, the three percentage divergence values are: $17.8 \%$ (maximum), $0.43 \%$ (minimum) and $8.6 \%$ (average).
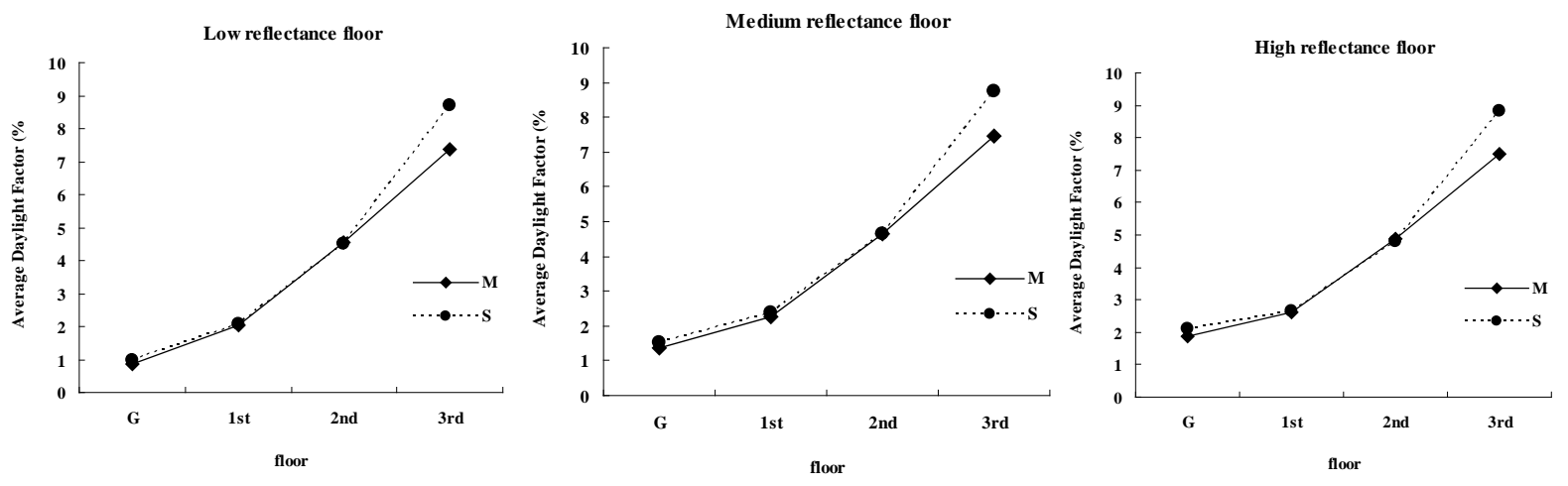

Fig. 4. Comparison of measured (M) and simulated (S) average daylight factors at each floor in a four-story atrium model without balconies.

Overall, the varying trends of the simulated data are similar to those of the measured data in the atrium models with various facades and well floor reflectances. The trends express an exponential form from the atrium base to the top. For all the models studied, the average percentage difference between simulation and measurement was less than $10 \%$ even though a few positions see a relatively larger divergence. The bigger disagreements between the measurement and simulation can be explained by the geometric and photometric deviations between the physical model and the Radiance model, which have been analysed in two earlier papers $[9,10]$. In addition, the nature of some Radiance algorithms also contributed to the divergence brought by the reflected light from the lighter surfaces. In general, the Radiance simulations have been validated by the measurements in this study. 


\section{Daylighting performance in an atrium}

Radiance was used in this section to calculate the average daylight factor in adjoining rooms of an atrium model. The ADF was used as an indicator of fundamental daylight performance in rooms of atria under CIE overcast sky conditions.

\subsection{Atrium model}
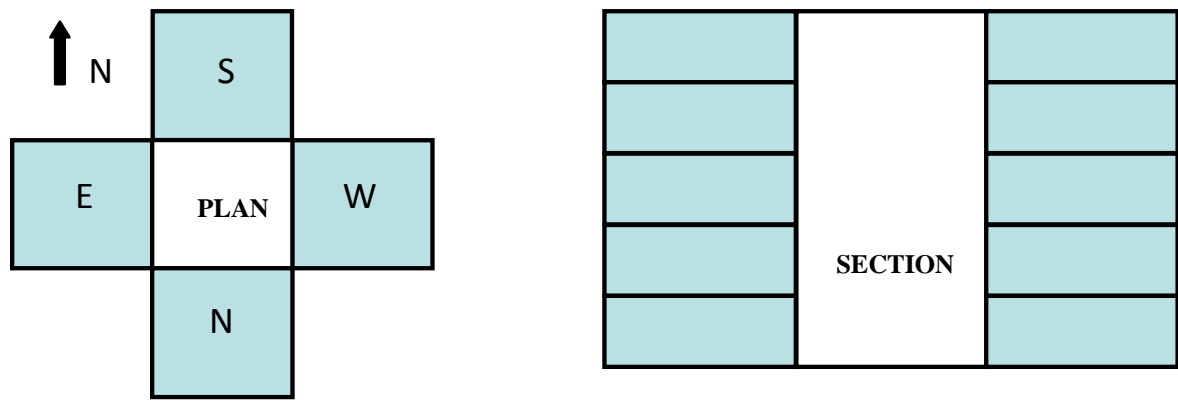

Fig. 5. Plan and section of a five-story atrium model (dark part: rooms; white part: well).

The atrium model studied consisted of a five-story building with a centre square well and four-sided rooms with the same size (Fig. 5), where N, E, S and W indicate the direction the well opening is facing. The WI value of the atrium model was 1.55 , which means a medium atrium. The solid part of the well (including well floor and balcony) had reflectance values of $0,0.2,0.4,0.6$ and 0.8 . The rooms had a fixed ceiling reflectance $(0.8)$, wall reflectance $(0.5)$ and floor reflectance (0.25). In addition, the models were divided into three groups in terms of the façade/balcony type - see Fig. 6 where (a) = window with no balcony (opening façade); (b) $=1 / 4$ room height balcony; (c) $=1 / 2$ room height balcony.

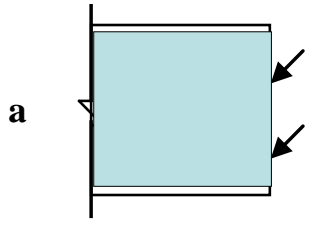

b

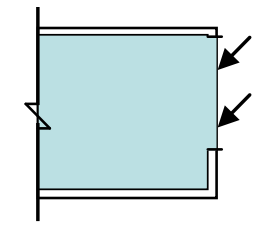

c

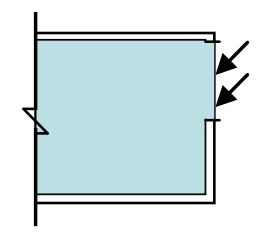

Fig. 6. Three different types of façade.

\subsection{Average daylight factors in rooms}

The rooms at the top floor ( $4^{\text {th }}$ floor), middle floor $\left(2^{\text {nd }}\right.$ floor) and ground floor were studied. Fig. 7 shows the variations of ADF in rooms at the three different heights in the atrium with various well reflectances and façade systems. Apparently, the higher balcony reduces the incident light for the rooms, giving a lower ADF. The 1/2 balcony room had the least daylight. At different floors, the daylight levels in the $1 / 4$ balcony room are close to those in the room with no balcony, which are much larger than those of $1 / 2$ balcony room. Interestingly, when the positions of room become lower, the differences between curves of the $1 / 2$ balcony and the curves of the open window tended to be smaller. This implies that the impact of the balcony on ADF is decreasing near the base. On the top floor, the ADF values of rooms are not significantly influenced by the increasing well solid surface reflectances. The middle floor and ground floor, nevertheless, see that an increasing well surface increases the ADF in rooms. The lower are the floors then the slopes of the curves (reflectance as a function) are steeper. This means increased magnitudes of ADF by increasing reflectance tend to be larger at lower floors than those at upper floors, especially for the model with $1 / 2$ balcony. For instance, the relative differences between refl0 and refl0.8 of the open window and $1 / 2$ balcony on the top floor are $2 \%$ and $12 \%$ respectively, whilst the two values have greatly increased to $73 \%$ and 
$188 \%$ on the ground floor. This might be due to the fact that the main components of ADF consist of reflected light. For all models, the rooms on the top floor have the largest daylight level which decays in an exponential form toward the atrium ground (see the sub-figure at the right of the below part in Fig.7). This responds with the results of scale model measurement in section 2 .
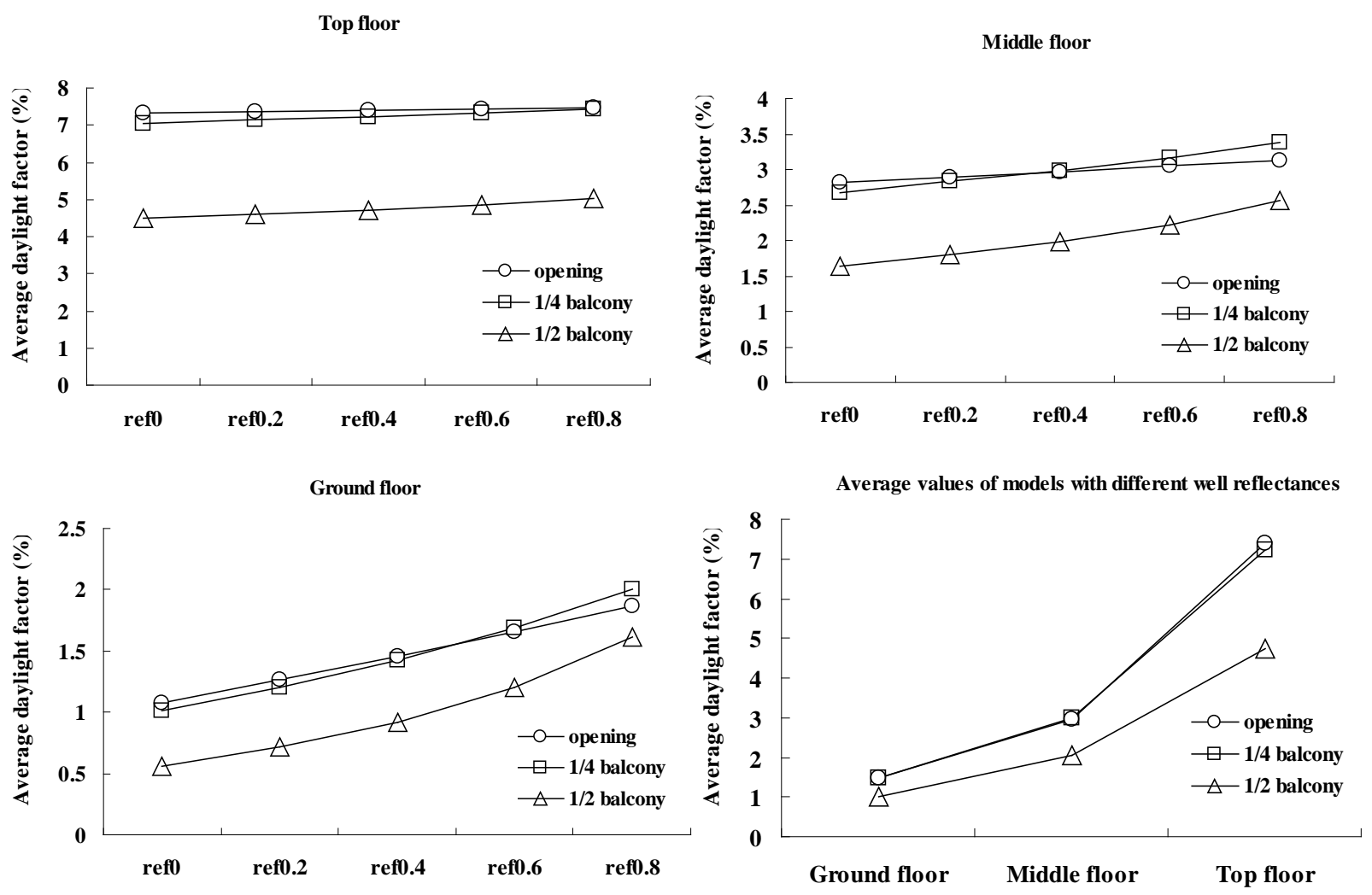

Fig. 7. Variations of ADF in rooms of different floors in an atrium with various façades and well surface reflectance.

\section{Energy performance in an atrium}

DaySim (using basic Radiance algorithm) [11, 12] was utilized here to predict the annual electrical lighting energy usage in adjoining rooms of the atrium model. The annual lighting consumption per unit area was used for the assessment of fundamental lighting energy performance in the daylit adjoining rooms. Based on the weather datasets, Daysim can derive the sky conditions include a large range of sky models like overcast sky, intermediate sky and clear sky [11].

\subsection{Simulation preparations}

The atrium model studied for energy analysis was the same as the model in section 3.1. The location of the atrium was in Sheffield, UK (Lat: 53.50'N; Lon: 1.00'E). According to Fig.5, the rooms at different sides can be named as: east-facing room (e), west-facing room (w), south-facing room (s) and north-facing room (n). The reflectances of room components were still kept the same while only a medium value 0.4 was adopted as the well solid surface reflectance. The facades followed the three types shown in Fig.6.

For lighting energy calculation, several fundamental aspects [12] were quoted as follows:

(1) Daylight savings time is from April 1st to October $31^{\text {st }}$; (2) The atrium is in an office building. All adjoining spaces are used as the working zone, which is occupied Monday through Friday from 8:00 AM to 17:00 PM; (3) A minimum illuminance level of 500 lux is 
needed to support the working task of occupants; (4) The electric lighting system has been installed in the adjoining spaces with a typical lighting power density of $14.00 \mathrm{~W} / \mathrm{m}^{2}$; (5) The adjoining rooms do not use any dynamic shading device system and the lighting system is manually controlled with an on/off switch.

\subsection{Lighting energy usage in rooms}

Fig. 8 shows the annual electrical lighting energy consumptions (unit area) of rooms at the three different heights and four different sides in the atrium with various façade systems. It is obvious that generally the rooms on the higher floors have the lowest electricity load for the task illuminations in a whole year because the positions can receive the most incident daylight from the sky. The ground floor rooms are the ones which consume the most electrical lighting energy. Surprisingly, the lighting energy usage in rooms in the middle floor is just a little lower than the rooms on the ground floor while it is much larger than the top floor. Taking the east-facing side room as an example, the average annual lighting load at three different floors are $24.23 \mathrm{kWh} / \mathrm{m}^{2}$ (top), $30.87 \mathrm{kWh} / \mathrm{m}^{2}$ (middle) and $31.30 \mathrm{kWh} / \mathrm{m}^{2}$ (ground) respectively. Considering the energy algorithm in Daysim [11], the orientations of the rooms also have some impact on the lighting energy usage. The rooms facing south or east apparently consume the less energy $\left(28.76 \mathrm{kWh} / \mathrm{m}^{2}\right.$ or $\left.28.8 \mathrm{kWh} / \mathrm{m}^{2}\right)$ whilst the rooms facing west and north need more electricity for task lighting $\left(29.14\right.$ and $\left.29.23 \mathrm{kWh} / \mathrm{m}^{2}\right)$. Interestingly, the differences between rooms with 'good' orientation and rooms with 'bad' orientation are not very large. This might be explained by a fact that the building location (Sheffield, UK) is dominated by overcast sky conditions, which has a radial symmetric sky luminance distribution [12].
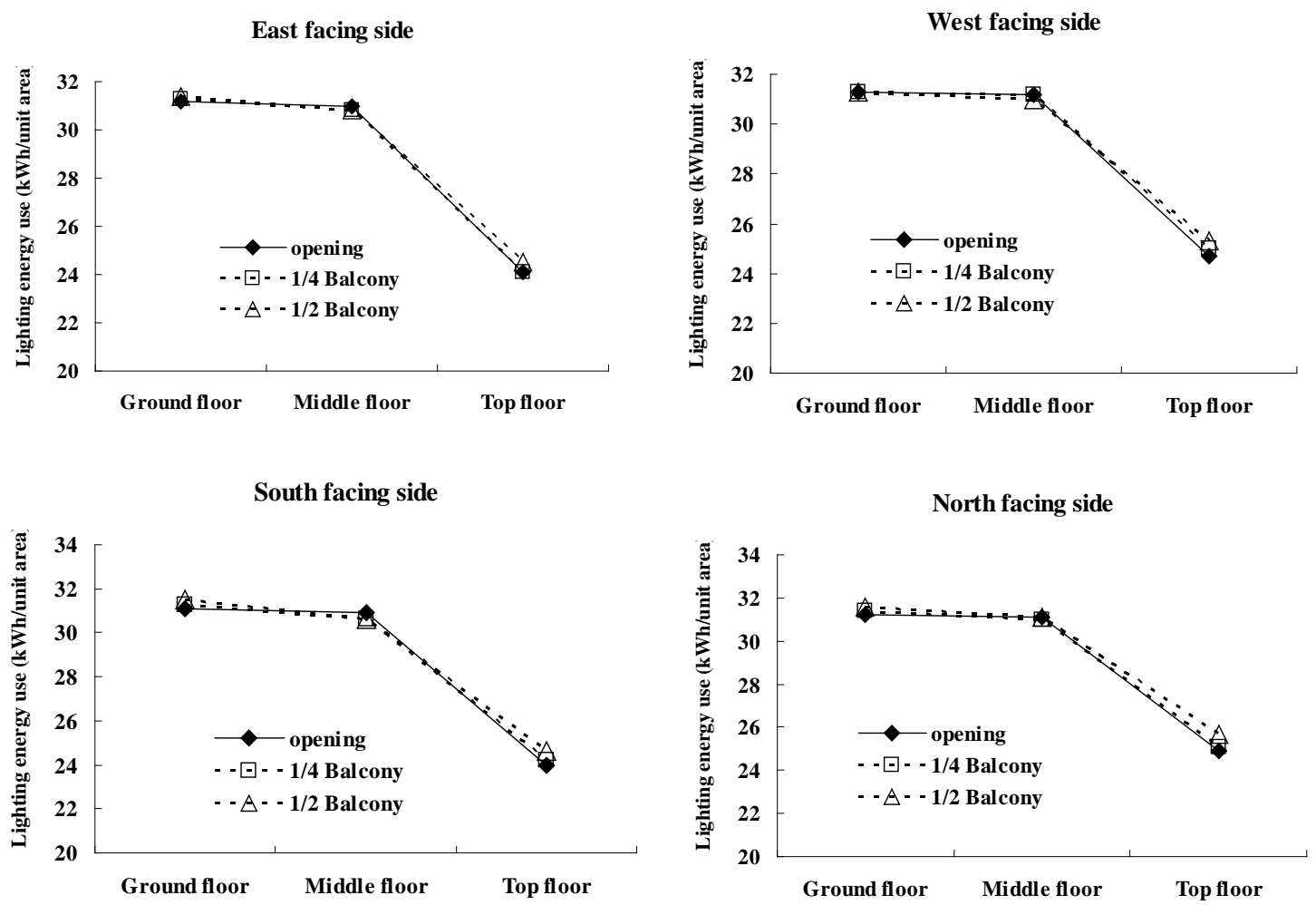

Fig. 8.Annual lighting energy consumptions $\left(\mathrm{kWh} / \mathrm{m}^{2}\right)$ of adjoining rooms at different floors and different sides in an atrium.

Table.1 expresses the effect of façade systems on the annual lighting energy consumption in adjoining rooms. For the top floor and the ground floor, the higher is the balcony then the more is the lighting energy usage. This actually coincided with the basic daylight performance 
(worst scenario: overcast sky) of rooms in section 3.2. However, the middle floor sees a different trend: the rooms with two different balconies have the same energy consumption which is less than the value of rooms with an open façade system. The trend displays a little difference from the variation of basic daylight performance (overcast sky) on this floor. It should be noted that the daylighting calculation used in the Daysim energy analysis is based on real weather dataset and the sunlight has also been included. Also, the daylight levels in the room of middle floor would be significantly influenced by the well wall, which can bring more reflected light into the rooms than other floors $[3,4]$. But, the average lighting consumption in all rooms with opening façade is $28.88 \mathrm{kWh} / \mathrm{m}^{2}$, while the values in all rooms with $1 / 4$ balcony and $1 / 2$ balcony are 28.94 and $29.12 \mathrm{kWh} / \mathrm{m}^{2}$ respectively. So, increasing the balcony height generally increases energy consumption if all the rooms of in the atrium are considered.

Table 1. Annual lighting energy consumptions $\left(\mathrm{kWh} / \mathrm{m}^{2}\right)$ and atria with various facade systems.

\begin{tabular}{|c|c|c|c|c|}
\hline \multirow{2}{*}{ Floor } & \multirow{2}{*}{ Side } & \multicolumn{3}{|c|}{ Annual lighting consumption $\left(\mathrm{kWh} / \mathrm{m}^{2}\right)$} \\
\hline & & opening & 1/4 balcony & $1 / 2$ balcony \\
\hline \multirow{5}{*}{$\begin{array}{l}\text { Ground } \\
\text { floor }\end{array}$} & $\mathrm{E}$ & 31.2 & 31.3 & 31.4 \\
\hline & W & 31.3 & 31.3 & 31.3 \\
\hline & $\mathrm{S}$ & 31.1 & 31.3 & 31.5 \\
\hline & $\mathrm{N}$ & 31.2 & 31.4 & 31.6 \\
\hline & $\begin{array}{l}4 \text { sides } \\
\text { average }\end{array}$ & 31.2 & 31.3 & 31.5 \\
\hline \multirow{5}{*}{$\begin{array}{l}\text { Middle } \\
\text { floor }\end{array}$} & $\mathrm{E}$ & 31 & 30.8 & 30.8 \\
\hline & W & 31.2 & 31.2 & 31 \\
\hline & $\mathrm{S}$ & 30.9 & 30.6 & 30.6 \\
\hline & $\mathrm{N}$ & 31.1 & 31 & 31.1 \\
\hline & $\begin{array}{l}4 \text { sides } \\
\text { average }\end{array}$ & 31.0 & 30.9 & 30.9 \\
\hline \multirow{5}{*}{$\begin{array}{l}\text { Top } \\
\text { floor }\end{array}$} & $\mathrm{E}$ & 24.1 & 24.1 & 24.5 \\
\hline & W & 24.7 & 25 & 25.3 \\
\hline & S & 24 & 24.2 & 24.6 \\
\hline & $\mathrm{N}$ & 24.9 & 25.1 & 25.7 \\
\hline & $\begin{array}{l}4 \text { sides } \\
\text { average }\end{array}$ & 24.4 & 24.6 & 25.0 \\
\hline
\end{tabular}

\section{Conclusions}

This study has given an assessment of daylight performance and artificial lighting consumption in adjoining spaces in an atrium model. Some findings can be drawn from the results and discussions above:

(1) There is an agreement between scale model measurements and Radiance simulations on average daylight levels of rooms in a medium height atrium. This enhances again the validation of basic Radiance algorithm in calculating atrium daylight levels.

(2) Under overcast sky conditions, the varying trend of average daylight levels in rooms expresses an exponential form along the vertical direction from the top to the base.

(3) Under overcast sky conditions, only the highest balcony can have a detrimental effect on the average daylight levels in rooms.

(4) The rooms on the top floor mainly receive direct sky light and are clearly not influenced by the variation of well reflectances; the rooms on the middle floor get both the direct 
daylight and reflected daylight; the rooms at ground floor level are substantially dependent on reflected daylight.

(5) In an atrium with four-sided adjoining rooms, the rooms on the top floor need the least energy for electrical lighting; the lighting energy performances are similar for the rooms at the middle floor and ground floor, both of which are much worse than the top floor.

(6) In an atrium with four-sided adjoining rooms located in the northern hemisphere, the rooms facing east and south perform better than the rooms facing north and south in terms of the lighting energy saving through daylight usage.

(7) In a four-sided atrium with adjoining rooms, the well facade consisting of the higher balconies and open windows would generally have a more negative impact on lighting energy saving in rooms than the open facades or facades with lower balconies (façade types in fig. 6). The limitations of the study are: (i) only the model with a square plan was analyzed, whose four sides were the same length; (ii) the façade configurations were relatively simple and no glazing has been considered in the models; (iii) the energy calculations are just focused on a site with a simple cloudy sky condition with little sunlight involved. Future work will focus on models with a broader range of geometries, more complicated well facades and a variety of sky luminance conditions at typical climate zones around the world.

\section{References}

[1] M. Aizlewood, The daylighting of atria: a critical review, ASHRAE Transactions 101, 1995, pp. 841-857.

[2] S. Sharples and D. Lash, Daylight in atrium buildings: a critical review, Architectural Science Review 50, 2007, pp. 301-312.

[3] P. Littlefair, Daylight prediction in atrium buildings, Solar Energy 73, 2002, pp. 105-109.

[4] J. Du and S. Sharples, Computational simulations for predicting vertical daylight levels in atrium buildings, Proc. of International Building Performance Simulation Association Conference, 2009, pp. 272-279.

[5] R. J. Cole, The effect of the surfaces adjoining atria on the daylight in adjacent spaces, Building and Environment 25, 1990, pp. 37-42.

[6] Ø. Aschehoug, Daylight in glazed spaces, Building Research \& Information 20, 1992, pp. 242-245.

[7] B. Calcagni and M. Paroncini, Daylight factor prediction in atria building designs, Solar Energy 76, 2004, pp. 669-682.

[8] M. R. Atif and A. D. Galasiu, Energy performance of daylight-linked automatic lighting control systems in large atrium spaces: report on two field-monitored case studies, Energy and Buildings 35, 2003, pp. 441-461.

[9] M. Aizlewood, K. Isaac, P. Littlefair, A scale model study of daylighting in atrium buildings, Proc. of the IESANZ, 1996.

[10]M. Aizlewood, J. Butt, K. Isaac, P. Littlefair, Daylight in atria: a comparison of measurement, theory and simulation, Proc. of Lux Europa, 1997.

[11]C. F. Reinhart, S. Herkel, The simulation of annual daylight illuminance distributions - a state-of-the-art comparison of six Radiance-based methods, Energy and Buildings 32, 2000, pp. 167-187.

[12]C. F. Reinhart, Tutorial on the use of Daysim simulations for sustainable design, IRCNRCC, 2006. 